\title{
Paleoambiente en cuatro sitios mexicanos del Pleistoceno tardío con actividad humana inferidos a partir de la fauna
}

Víctor Adrián Pérez-Grespo, Joaquín Arroyo-Cabrales, Pedro Morales-Puente, Patricia Ochoa-Castillo

\section{Víctor Adrián Pérez-Grespo}

vapc79@gmail.com

Pedro Morales-Puente

Instituto de Geología, Universidad Nacional Autónoma de México, Circuito de la Investigación Científica S/N, Ciudad Universitaria, Coyoacán 04150, CDMX, México.

\section{Joaquín Arroyo-Gabrales}

Laboratorio de Arqueozoología 'M. en C. Ticul Álvarez Solórzano', Subdirección de Laboratorios y Apoyo Académico, INAH, Moneda 16 Col. Centro, Cuauhtémoc 06060, CDMX, México.

Pedro Morales-Puente

Laboratorio de Isotopos Estables-LANGEMInstituto de Geología, Universidad Nacional Autónoma de México, Circuito de la Investigación Científica S/N, Ciudad Universitaria, Coyoacán 04150, CDMX, México.

\section{Patricia Ochoa-Gastillo}

Subdirección de Arqueología, Museo Nacional de Antropología, INAH, Av. Paseo de la Reforma y Calzada Gandhi, Col. Chapultepec Polanco, Miguel Hidalgo 11560, CDMX, México.
BOL. SOC. GEOL. MEX. 2019

VOL. 71 NO. 2

P. $343-358$

http://dx.doi.org/10.18268/BSGM2019v7 ln2a7

\section{RESUMEN}

El ambiente que existió en cuatro sitios mexicanos del Pleistoceno tardío, con presencia humana, fue inferido a partir de las relaciones isotópicas de carbono y oxígeno presentes en el esmalte de los herbívoros que habitaron estos sitios y que fueron obtenidos de estudios previos. Los análisis indican la presencia de bosques con pastizales en los sitios Fin del Mundo, Rancho La Amapola y Valsequillo, mientras que Tocuila contaba con pastizales y condiciones más húmedas que las actuales. En los cuatros sitios, estos ambientes favorecieron la coexistencia de diversas especies de herbívoros y permitieron el establecimiento de los primeros pobladores de México.

Palabras clave: Paleoambiente, primeros pobladores, Pleistoceno tardío, isotopos estables.

\section{ABSTRACT}

The environment of four late Pleistocene Mexican sites, with human presence, was inferred using carbon and oxygen stable isotope relationships from herbivores enamel, which lived at those sites and was obtained from previous studies. Those analyses showed the presence of forest with grasslands at the sites Fin del Mundo, Rancho La Amapola, and Valsequillo, while Tocuila had grasslands and more humid environmental conditions than at present. At the four localities, these environments favored the coexistence of several herbivorous species and allowed the establishment of the first people in Mexico.

Keyzords: Paleoenvironment, early settlers, late Pleistocene, stable isotopes. 


\section{Introducción}

Uno de los aspectos importantes de los estudios arqueológicos es inferir las condiciones ambientales que existieron en un sitio cuando éste fue ocupado por un grupo humano en el pasado (Grayson, 1981). Para este fin, existen diversas metodologías tales como los estudios y/o análisis geomorfológicos, de paleosuelos, polínicos, faunísticos e isotópicos en los elementos óseos (Garrison, 2016; Hart, 2016; Neumann et al., 2017; Casar et al., 2017; Lyman, 2017). Estos últimos se basan en cuantificar las relaciones isotópicas de elementos como carbono y nitrógeno en el colágeno del hueso y de carbono y oxígeno en el esmalte dental y han sido realizados principalmente en mamíferos. Estos análisis permiten inferir la dieta, el hábitat y aspectos fisiológicos de las especies halladas en un sitio, pero además, de manera indirecta, el tipo de vegetación y las condiciones climáticas que existieron en dicha localidad (Koch, 1998; Lee-Throp y Sponheimer, 2007).

En México existen algunos sitios arqueo-paleontológicos pertenecientes al Pleistoceno tardío, donde se han hallado evidencias de actividad humana de este lapso de tiempo. Dichos vestigios pueden ser artefactos líticos, que podrían estar asociados a restos de fauna de esta época, e inclusive, existen huesos de estos animales que tienen evidencia de modificación antropogénica (Morett et al., 1998; Ochoa-Castillo et al., 2003; Mirambell, 2012; Sánchez et al., 2014; Chatters et al., 2016; Sánchez y Carpenter, 2016).

A pesar de que en algunos de estos sitios se han realizado estudios arqueozoológicos, son pocos los sitios arqueo-paleontológicos mexicanos donde se han efectuado análisis isotópicos de la mastofauna presente. De hecho, los trabajos previos solo se han enfocado a inferir la dieta y el hábitat de los animales hallados y han omitido discurrir el posible ambiente que existió en los lugares y que posibilitaron la ocupación humana (Pérez-Crespo et al., 2014, 2018). Por lo anterior, en el presente artículo se infiere el paleoambiente de cuatro sitios mexicanos del Pleistoceno tardío con ocupa- ción humana (Fin del Mundo, Sonora; Rancho La Amapola, San Luis Potosí; Tocuila, Estado de México y Valsequillo, Puebla) usando las relaciones isotópicas de carbono y oxígeno presentes en el esmalte dental u osteodentina de la mastofauna hallada en éstos.

\subsection{ISÓTOPOS ESTABLES DE GARBONO Y OXÍGENO}

En la naturaleza existen diversos reservorios del elemento carbono (Koch, 1998). Sin embargo, los animales no son capaces de incorporarlo de manera directa, por lo que este elemento es fijado a partir del $\mathrm{CO}_{2}$ atmosférico a través de la fotosíntesis de la cual existen tres vías en las plantas. La primera vía fotosintética o Ciclo Calvin-Benson se encuentra en árboles y arbustos y algunos pastos de zonas frías. En este proceso, la primera molécula producida posee una estructura de tres carbonos, por lo que también se denomina vía $\mathrm{C}_{3}$, la cual es fijada por la rubisco 1,5-bifosfato carboxilasa oxigenasa (Medrano y Flexas, 2000). Los valores isotópicos de carbono de plantas con metabolismo $\mathrm{C}_{3}$ van de -35\% a -22\%o (O’Leary, 1981; van der Merwe y Medina, 1989; Drucker y Bocherens, 2009).

La segunda vía fotosintética, llamada HatchSlack, tiene características que la hacen diferente de la $\mathrm{C}_{3}$. Una de ellas es la presencia de una molécula de cuatro carbonos la cual es fijada por la fosfoenolpiruvato carboxilasa, por lo que se le conoce también como vía $\mathrm{C}_{4}$ (Medrano y Flexas, 2000). Además, las plantas que poseen este tipo de fotosíntesis presentan dos tipos de células en sus hojas donde se lleva a cabo el proceso fotosintético: del mesófilo y de la vaina. Este metabolismo se encuentra en pastos y algunas herbáceas de las familias Asteraceae y Quenopodiaceae, así como en algunos árboles y arbustos de zonas cálidas. Los valores isotópicos de carbono se encuentran en el rango de $-14 \%$ a $-12 \%$ (Ehleringer y Monson, 1993; Cerling, 1999; Cerling y Ehleringer, 2000). La última vía fotosintética se encuentra en plantas suculentas como cactáceas, orquídeas y bromelias. Se denomina Metabolismo Ácido Crasuláceo, CAM por sus siglas en inglés, ya que fue descu- 
bierto en especies de la familia Crassulaceae y se distinguen de las plantas $\mathrm{C}_{3}$ y $\mathrm{C}_{4}$ por poseer una anatomía suculenta (Andrade et al., 2007). Los valores de $\delta^{13} \mathrm{C}$ que posee el metabolismo CAM se encuentran entre $-35 \%$ y $-14 \%$, por lo que se hacen indistinguibles de las otras dos formas fotosintéticas (Gröcker, 1997; Andrade et al., 2007).

El carbono ingresa a los animales cuando las plantas son consumidas y se incorpora a sus estructuras y tejidos. Así los animales mostrarán valores de $\delta^{13} \mathrm{C}$ semejantes a la planta que consumieron, pero sufrirán un incremento con respecto a la fuente consumida debido a las reacciones bioquímicas que se producen por los diversos procesos metabólicos de los animales. En el caso del esmalte dental este incremento es de $14.1 \%$ por lo que animales que consuman plantas $\mathrm{C}_{3}$ tendrán valores de $\delta^{13} \mathrm{C}$ de $-19 \%$ a $-9 \%$; aquellos que se alimenten de plantas $\mathrm{C}_{4}$ mostrarán valores isotópicos de -2 $\%$ a $2 \%$, mientras que consumidores de ambos tipos de plantas tendrán rangos de $-9 \%$ a $-2 \%$ (MacFadden y Cerling, 1996).

El oxígeno, en cambio, ingresa a los mamíferos principalmente por el agua ingerida directamente y en menor cantidad a través del agua contenida en los alimentos y el inhalado por la respiración. Lo anterior se equilibra con el oxígeno que se pierde por la exhalación, el sudor, la orina y las heces. En el caso de los mamíferos, este balance se halla controlado por la temperatura corporal que en este grupo es constante y por lo mismo, los valores de $\delta^{18} \mathrm{O}$ de cualquier elemento óseo de estos animales serán similares entre si (Koch, 1998; Sánchez, 2005).

$\mathrm{El}$ agua que se ingiere proviene de la lluvia, cuyos valores isotópicos de oxígeno son afectados por diversos factores. Dentro de éstos, el principal es la temperatura ya que a mayores temperaturas los valores serán más positivos (Dansgaard, 1964; Castillo et al., 1985). La distancia de una fuente de agua con respecto al océano, la latitud, la altitud y la cantidad de lluvia afectan también las relaciones isotópicas del agua en una localidad (Castillo et al., 1985). Adicionalmente, algunas especies de herbí- voros que beben agua frecuentemente poseen valores de $\delta^{18} \mathrm{O}$ más bajos que aquellos que obtienen el agua de las plantas que consumen debido a que las hojas pierden agua por evapotranspiración y por lo tanto se presenta un enriquecimiento de ${ }^{18} \mathrm{O}$ en las mismas (Ambrose y DeNiro, 1986; Harris y Cerling, 2002).

\section{Lugares de estudio}

A continuación se describen los sitios de estudio (Figura 1).

\subsection{FIN DEL MUNDO}

Se ubica a $100 \mathrm{~km}$ al noroeste de Hermosillo, Sonora, a $633 \mathrm{msnm}$, en las coordenadas $29^{\circ} 45^{\prime} 29^{\prime \prime}$ $\mathrm{N}$ y $111^{\circ} 40^{\prime} 46^{\prime \prime} \mathrm{O}$. El sitio se encuentra en un sistema fluvial el cual ha expuesto una serie de islas en su cauce. En una de estas islas, denominada localidad 1, se han encontrado restos de gonfoterios (Cuvieronius sp.), mastodontes (Mammut americanum) y tapires (Tapirus sp.), por lo menos 20 lascas, dos ornamentos de huesos y cuatro puntas Clovis, así como fragmentos de carbón, uno de los cuales fue fechado en $11550 \pm 70$ años AP (13390 años calendario) (Sánchez et al., 2014).

\subsection{RANGHO LA AMAPOLA}

Se encuentra $7.8 \mathrm{~km}$ de Matehuala, en el estado de San Luis Potosí, a 1700 msnm, en las coordenadas $23^{\circ} 49^{\prime} \mathrm{N}, 100^{\circ} 43^{\prime} \mathrm{O}$. En este sitio se han encontrado restos de berrendos del Pleistoceno (Capromeryx mexicana), bisontes (Bison sp.), caballos (Equus cedralensis, E. conversidens, E. mexicanus), camellos (Camelops hesternus), gliptodontes (Glyptotherium sp.), llamas (Hemiauchenia macrocephala, H. vera, Hemiauchenia sp.), jabalíes (Platygonus sp.), mastodontes, mamuts (Mammuthus columbi), megalónidos (Megalonyx cf. M.jeffersoni), notroterios (Nothrotheriops shastensis), perezosos terrestres (Paramylodon harlanii), tapires (Tapirus haysii), así como micromamíferos y 


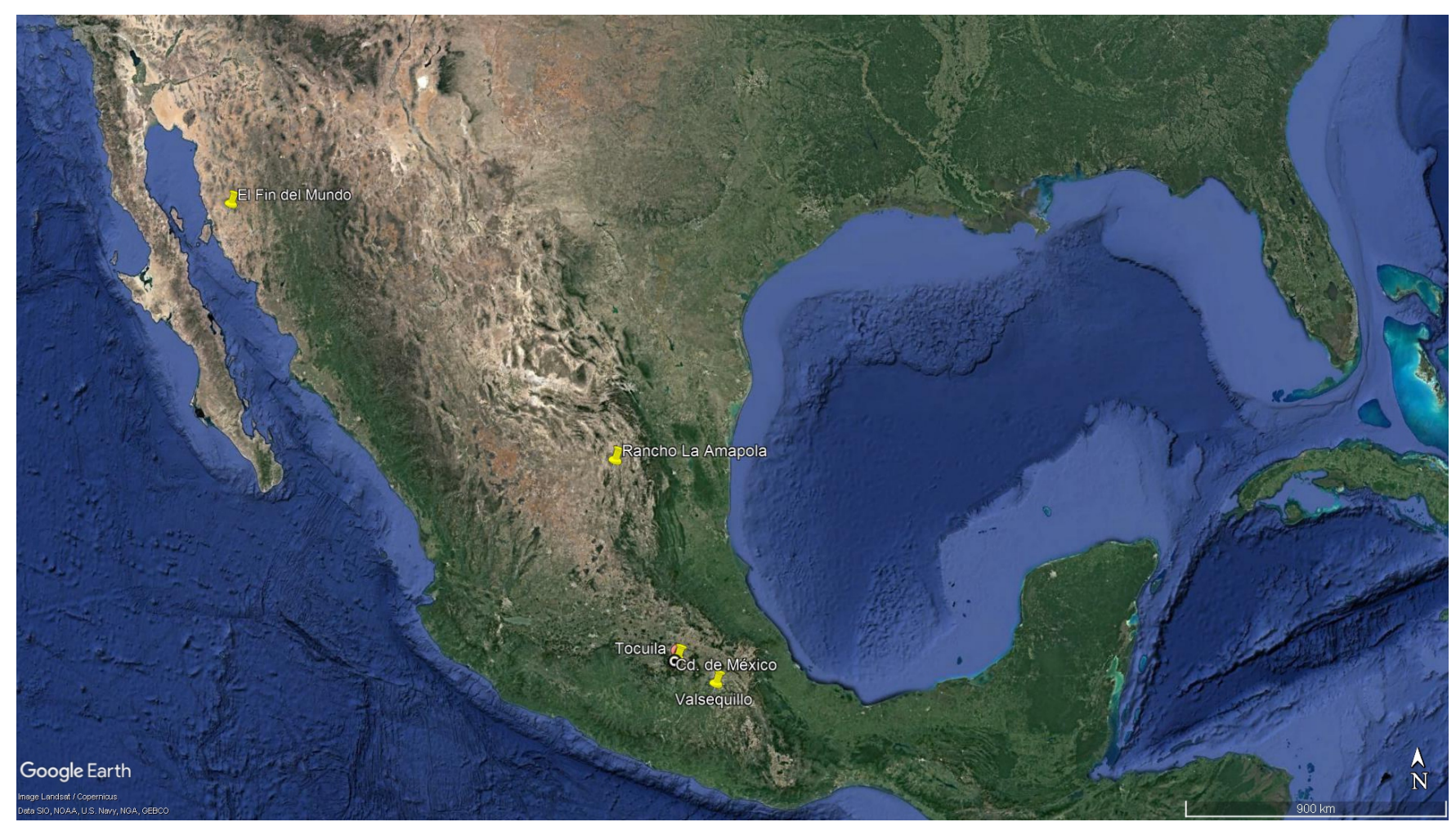

Figura 1 Sitios arqueo-paleontológicos de estudio.

otros vertebrados (Álvarez et al., 2012). El sitio ha sido fechado entre 33300 y 2480 años AP (Arroyo-Cabrales et al., 2010).

\subsection{TOGUILA}

Se localiza en el municipio de Texcoco, en el Estado de México, a 2240 msnm, en las coordenadas $19^{\circ} 31^{\prime} \mathrm{N}, 98^{\circ} 54^{\prime} \mathrm{O}$. En el sitio se han hallado restos de bisontes, caballos (Equus sp.), camellos, mamuts, felinos, liebres, ratones y aves, así como rastros de actividad humana. Ha sido fechado en $11188 \pm 70$ años AP (Morett et al., 1998).

\subsection{VALSEQUILLO}

Se encuentra $15 \mathrm{~km}$ al sur de la ciudad de Puebla, Puebla a $2040 \mathrm{msnm}$, en las coordenadas $18^{\circ}$ $54^{\prime} \mathrm{N}, 98^{\circ} 10^{\prime} \mathrm{O}$ (Cruz-Muñoz et al., 2009). En la zona se han encontrado rastros de actividad humana y restos fósiles de berrendos pleistocénicos (Tetrameryx sp.), bisontes, carpinchos (Neochoerus sp.), caballos, camellos, coyotes, gonfoterios (Stegomastodon sp.), gliptodontes, jabalíes (Platygonus compresus), llamas, mamuts, osos de cara corta (Arctodus simus), pampaterios (Pampatherium mexicanum), perezosos terrestres, así como diversas especies de carnívoros, lagomorfos y roedores. El sitio ha sido fechado en 20780 años AP. (Arroyo-Cabrales et al., 2010).

\section{Análisis de datos}

Se obtuvieron los valores isotópicos de carbono y oxígeno reportados por Pérez-Crespo et al. (2010, 2014, 2016, 2018) para las mastofaunas herbívoras del Fin del Mundo, Rancho La Amapola, Tocuila y Valsequillo. Los valores de $\delta^{13} \mathrm{C}$ fueron comparados con los reportados por MacFadden y Cerling (1996) para inferir el tipo de dieta (Tabla 1). Asimismo, los valores isotópicos de carbono y oxígeno fueron graficados siguiendo la propuesta de Feranec y MacFadden (2006), quienes indican que los valores de $\delta^{13} \mathrm{C}$ y $\delta^{18} \mathrm{O}$ negativos corres- 
Tabla 1. Valores isotópicos de carbono y oxígeno de la mastofauna del Fin del Mundo, Rancho La Amapola, Tocuila y Valsequillo.

\begin{tabular}{|c|c|c|c|c|}
\hline Especie & $\delta^{13} \mathrm{C}$ & $\delta^{18} \mathrm{O}$ & Dieta & Localidad \\
\hline Bison sp. & -1.3 & -6.8 & $\mathrm{C}_{4}$ & Rancho La Amapola \\
\hline Bison sp. & -1.3 & -6.6 & $\mathrm{C}_{4}$ & Rancho La Amapola \\
\hline Bison sp. & -3.2 & -7.4 & $\mathrm{C}_{3} / \mathrm{C}_{4}$ & Rancho La Amapola \\
\hline Bison sp. & -1.2 & -5.5 & $\mathrm{C}_{4}$ & Rancho La Amapola \\
\hline Camelops hesternus & -9.3 & -5.6 & $\mathrm{C}_{3}$ & Rancho La Amapola \\
\hline Camelops hesternus & -9.3 & -4.6 & $\mathrm{C}_{3}$ & Rancho La Amapola \\
\hline Camelops hesternus & -8.8 & -5.9 & $\mathrm{C}_{3} / \mathrm{C}_{4}$ & Rancho La Amapola \\
\hline Capromeryx mexicana & -5.7 & -4.6 & $\mathrm{C}_{3} / \mathrm{C}_{4}$ & Rancho La Amapola \\
\hline Capromeryx mexicana & -8.8 & -4.4 & $\mathrm{C}_{3} / \mathrm{C}_{4}$ & Rancho La Amapola \\
\hline Cuvieronius sp. & -4.4 & -5.2 & $\mathrm{C}_{3} / \mathrm{C}_{4}$ & Fin del Mundo \\
\hline Cuvieronius sp. & -4.2 & -5.4 & $\mathrm{C}_{3} / \mathrm{C}_{4}$ & Fin del Mundo \\
\hline Equus sp. & -0.5 & -7.4 & $\mathrm{C}_{4}$ & Valsequillo \\
\hline Equus sp. & -1.4 & -6.6 & $\mathrm{C}_{4}$ & Valsequillo \\
\hline Equus sp. & -0.5 & -7.4 & $\mathrm{C}_{4}$ & Valsequillo \\
\hline Equus sp. & -1.4 & -6.6 & $\mathrm{C}_{4}$ & Valsequillo \\
\hline Equus cedralensis & -2.7 & -3.6 & $\mathrm{C}_{3} / \mathrm{C}_{4}$ & Rancho La Amapola \\
\hline Equus cedralensis & -1.5 & -2.4 & $\mathrm{C}_{4}$ & Rancho La Amapola \\
\hline Equus cedralensis & -2.1 & -2.4 & $\mathrm{C}_{3} / \mathrm{C}_{4}$ & Rancho La Amapola \\
\hline Equus cedralensis & -3 & -1.6 & $\mathrm{C}_{3} / \mathrm{C}_{4}$ & Rancho La Amapola \\
\hline Equus cedralensis & -4.4 & -1.4 & $\mathrm{C}_{3} / \mathrm{C}_{4}$ & Rancho La Amapola \\
\hline Equus conversidens & -1.9 & -1.8 & $\mathrm{C}_{3} / \mathrm{C}_{4}$ & Rancho La Amapola \\
\hline Equus conversidens & -2.6 & -2.9 & $\mathrm{C}_{3} / \mathrm{C}_{4}$ & Rancho La Amapola \\
\hline Equus conversidens & -1.7 & -4.8 & $\mathrm{C}_{4}$ & Rancho La Amapola \\
\hline Equus mexicanus & -4.4 & -1.4 & $\mathrm{C}_{3} / \mathrm{C}_{4}$ & Rancho La Amapola \\
\hline Equus mexicanus & -5.9 & -2.4 & $\mathrm{C}_{3} / \mathrm{C}_{4}$ & Rancho La Amapola \\
\hline Equus mexicanus & -4.7 & -4 & $\mathrm{C}_{3} / \mathrm{C}_{4}$ & Rancho La Amapola \\
\hline Equus mexicanus & -6.9 & -3.2 & $\mathrm{C}_{3} / \mathrm{C}_{4}$ & Rancho La Amapola \\
\hline Equus mexicanus & -4.3 & -3.6 & $\mathrm{C}_{3} / \mathrm{C}_{4}$ & Rancho La Amapola \\
\hline Equus mexicanus & -5.1 & -1.9 & $\mathrm{C}_{3} / \mathrm{C}_{4}$ & Rancho La Amapola \\
\hline Equus mexicanus & -2.7 & -4 & $\mathrm{C}_{3} / \mathrm{C}_{4}$ & Rancho La Amapola \\
\hline Glypthotherium sp. & -4.6 & -5.8 & $\mathrm{C}_{3} / \mathrm{C}_{4}$ & Rancho La Amapola \\
\hline Glypthotherium sp. & -3.7 & -7 & $\mathrm{C}_{3} / \mathrm{C}_{4}$ & Rancho La Amapola \\
\hline Glypthotherium sp. & -4.5 & -7.6 & $\mathrm{C}_{3} / \mathrm{C}_{4}$ & Rancho La Amapola \\
\hline Hemiauchenia sp. & -10.3 & -5.6 & $\mathrm{C}_{3}$ & Rancho La Amapola \\
\hline Hemiauchenia sp. & -8 & -6.1 & $\mathrm{C}_{3} / \mathrm{C}_{4}$ & Rancho La Amapola \\
\hline Hemiauchenia macrocephala & -5.6 & -7.2 & $\mathrm{C}_{3} / \mathrm{C}_{4}$ & Rancho La Amapola \\
\hline
\end{tabular}


Tabla 1. Valores isotópicos de carbono y oxígeno de la mastofauna del Fin del Mundo, Rancho La Amapola, Tocuila y Valsequillo. (Continuación):

\begin{tabular}{|c|c|c|c|c|}
\hline Especie & $\delta^{13} \mathrm{C}$ & $\delta^{18} \mathrm{O}$ & Dieta & Localidad \\
\hline Hemiauchenia vera & -9.5 & -4.5 & $\mathrm{C}_{3}$ & Rancho La Amapola \\
\hline Mammut americanum & -8.7 & -3.9 & $\mathrm{C}_{3} / \mathrm{C}_{4}$ & Rancho La Amapola \\
\hline Mammut americanum & -7.2 & -4.7 & $\mathrm{C}_{3} / \mathrm{C}_{4}$ & Rancho La Amapola \\
\hline Mammut americanum & -9.2 & -4.6 & $\mathrm{C}_{3}$ & Rancho La Amapola \\
\hline Mammut americanum & -9 & -4.9 & $\mathrm{C}_{3}$ & Fin del Mundo \\
\hline Mammut americanum & -9 & -4.9 & $\mathrm{C}_{3}$ & Fin del Mundo \\
\hline Mammut americanum & -9.5 & -4.6 & $\mathrm{C}_{3}$ & Fin del Mundo \\
\hline Mammut americanum & -9.5 & -5.6 & $\mathrm{C}_{3}$ & Fin del Mundo \\
\hline Mammut americanum & -9.8 & -5.4 & $\mathrm{C}_{3}$ & Fin del Mundo \\
\hline Mammuthus columbi & -3.8 & -5.1 & $\mathrm{C}_{3} / \mathrm{C}_{4}$ & Rancho La Amapola \\
\hline Mammuthus columbi & -1.8 & -4.4 & $\mathrm{C}_{4}$ & Rancho La Amapola \\
\hline Mammuthus columbi & -3.3 & -3.8 & $\mathrm{C}_{3} / \mathrm{C}_{4}$ & Rancho La Amapola \\
\hline Mammuthus columbi & -0.9 & -4.5 & $\mathrm{C}_{4}$ & Rancho La Amapola \\
\hline Mammuthus columbi & -3.9 & -3.6 & $\mathrm{C}_{3} / \mathrm{C}_{4}$ & Tocuila \\
\hline Mammuthus columbi & -3.5 & -8.1 & $\mathrm{C}_{3} / \mathrm{C}_{4}$ & Tocuila \\
\hline Mammuthus columbi & -1.3 & -13.3 & $\mathrm{C}_{4}$ & Tocuila \\
\hline Mammuthus columbi & -4.4 & -5 & $\mathrm{C}_{3} / \mathrm{C}_{4}$ & Tocuila \\
\hline Mammuthus columbi & -5.1 & -5.5 & $\mathrm{C}_{3} / \mathrm{C}_{4}$ & Tocuila \\
\hline Mammuthus columbi & -0.9 & -4.7 & $\mathrm{C}_{4}$ & Valsequillo \\
\hline Mammuthus columbi & -2.6 & -7.5 & $\mathrm{C}_{3} / \mathrm{C}_{4}$ & Valsequillo \\
\hline Megalonyx cf. M. jeffersoni & -3.1 & -4.5 & $\mathrm{C}_{3} / \mathrm{C}_{4}$ & Rancho La Amapola \\
\hline Neochoerus sp. & -2.1 & -6.5 & $\mathrm{C}_{3} / \mathrm{C}_{4}$ & Valsequillo \\
\hline Nothroteriops shastensis & -9.8 & -3.8 & $\mathrm{C}_{3}$ & Rancho La Amapola \\
\hline Platygonus sp. & -9.1 & -4.8 & $\mathrm{C}_{3}$ & Rancho La Amapola \\
\hline Paramylodon harlani & -4.8 & -7.7 & $\mathrm{C}_{3} / \mathrm{C}_{4}$ & Rancho La Amapola \\
\hline Paramylodon harlani & -1.8 & -4.7 & $\mathrm{C}_{4}$ & Valsequillo \\
\hline Stegomastodon sp. & -3.2 & -4.7 & $\mathrm{C}_{3} / \mathrm{C}_{4}$ & Valsequillo \\
\hline Stegomastodon sp. & -4 & -4.7 & $\mathrm{C}_{3} / \mathrm{C}_{4}$ & Valsequillo \\
\hline Stegomastodon sp. & -4.3 & -8.6 & $\mathrm{C}_{3} / \mathrm{C}_{4}$ & Valsequillo \\
\hline Stegomastodon sp. & -4.4 & -9.5 & $\mathrm{C}_{3} / \mathrm{C}_{4}$ & Valsequillo \\
\hline Stegomastodon sp. & -4.8 & -8 & $\mathrm{C}_{3} / \mathrm{C}_{4}$ & Valsequillo \\
\hline Stegomastodon sp. & -4.5 & -7.7 & $\mathrm{C}_{3} / \mathrm{C}_{4}$ & Valsequillo \\
\hline Tapirus sp. & -10.3 & -4.7 & $\mathrm{C}_{3}$ & Fin del Mundo \\
\hline Tapirus haysii & -10.7 & -5.3 & $\mathrm{C}_{3}$ & Rancho La Amapola \\
\hline Tapirus haysii & -10.5 & -5.9 & $\mathrm{C}_{3}$ & Rancho La Amapola \\
\hline Tapirus haysii & -10.6 & -5.3 & $\mathrm{C}_{3}$ & Rancho La Amapola \\
\hline
\end{tabular}


ponden a zonas de vegetación cerrada, como bosques o selvas, y aquellos que son positivos, a zonas de vegetación abierta como pastizales o sabanas (Tabla 1; figuras 2, 3, 4 y 5).

\section{Resultados}

\subsection{FIN DEL MUNDO}

$\mathrm{El}$ valor promedio de $\delta^{13} \mathrm{C}$ para los gonfoterios del Fin del Mundo fue de $-4.3 \%$, con rango de valores de $-4.2 \%$ a $-4.4 \%$, mientras que para el oxígeno la media fue de $-5.3 \%$ o con un intervalo de $-5.2 \%$ o a $-5.4 \%$. En el caso de los mastodontes, el intervalo de valores de $\delta^{18} \mathrm{O}$ fue de $-5.6 \%$ a $-4.6 \%$ o con un promedio de $-5.1 \%$, mientras que para el carbono, éste fue de $-9.3 \%$ con un valor máximo de -9.8\% y uno mínimo de $-9.0 \%$. Para el tapir, sus valores de $\delta^{13} \mathrm{C}$ y $\delta^{18} \mathrm{O}$ fueron de - $10.3 \%$ y $-4.8 \%$ respectivamente (Tabla 1). Asimismo, la Figura 2 muestra que los mastodontes y el tapir del Fin del Mundo forman un grupo diferente y se ubican en el lado izquierdo del gráfico mientras que los gonfoterios se localizan en el lado derecho del mismo.

\subsection{RANCHO LA AMAPOLA}

En la Tabla 1 se pueden observar los valores promedios, así como los valores máximos y mínimos de carbono y oxígeno de cada una de las especies de mamíferos herbívoros hallados en Rancho La Amapola. Se destaca que los bisontes presentan en el valor isotópico promedio de carbono más positivo, $-1.1 \%$ mientras que los tapires exhibieron el valor de $\delta^{13} \mathrm{C}$ promedio más negativo, -10.6\% . En el caso del oxígeno, el caballo Equus cedralensis muestra el valor promedio más positivo, $-2.5 \%$, mientras que la llama Hemiuachenia macrocephala posee el más negativo, $-7.2 \%$.

La Figura 3 muestra que los bisontes, algunos caballos y el mamut se ubican a la derecha, lo cual

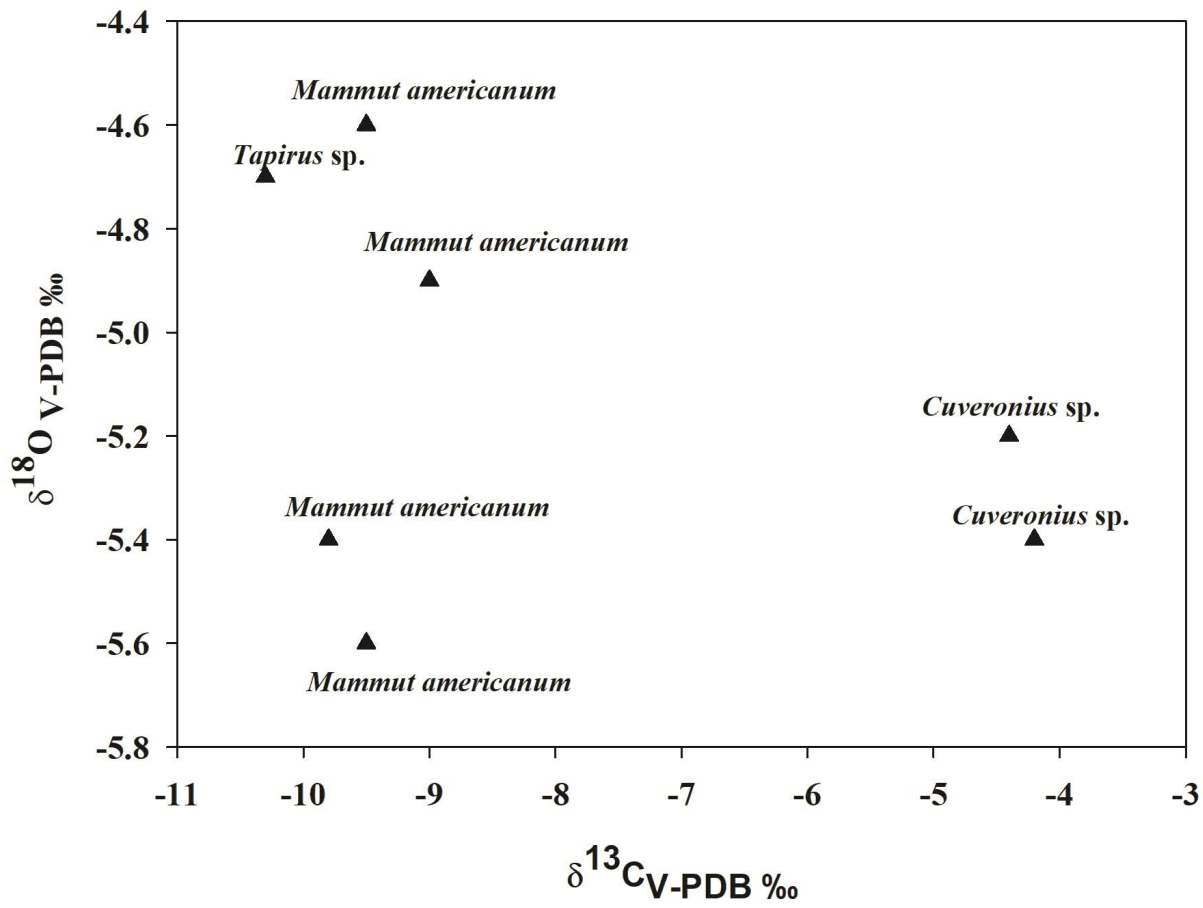

Figura 2 Comparación de los valores isotópicos de carbono y oxígeno de los herbívoros del Fin del Mundo. Los valores isotópicos de carbono y oxígeno están expresados en Vienna-Pee-Dee- Belemnite y en por mil (V-PDB \%o). 


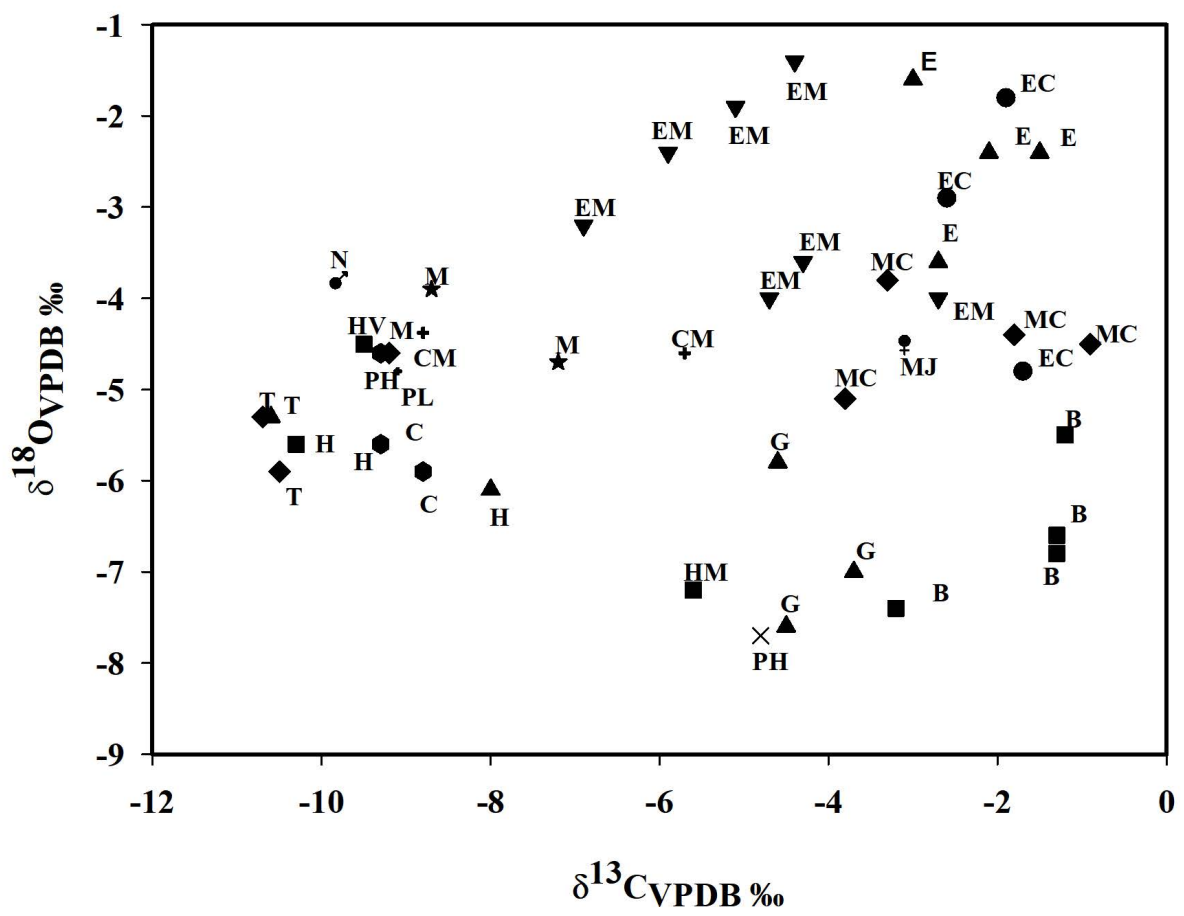

Figura 3 Comparación de los valores isotópicos de carbono y oxígeno de los herbívoros de Rancho La Amapola. Los valores isotópicos de carbono y oxígeno están expresados en Vienna-Pee-Dee-Belemnite y en por mil (V-PDB \%o). B: Bison sp., C: Camelops hesternus, CM: Capromeryx mexicana, E: Equus cedralensis, EC: Equus conversidens, EM: Equus mexicanus, G: Glyptotherium sp., H: Hemiauchenia sp., HM: Hemiauchenia macrocephala, HV: Hemiauchenia vera, M: Mammut americanum, MC: Mammuthus columbi, MJ: Megalonyx cf. M. jeffersoni, N: Notrotheriops shastensis, PH: Paramylodon harlani, PL: Platygonus sp., T: Tapirus haysii.

indica que estos animales vivieron en zonas de vegetación abierta. En el caso de los tapires, algunas llamas, camellos, mastodontes, jabalíes y el perezoso de Shasta se ubican a la izquierda, lo que significa que estos animales habitaron en zonas cerradas. Finalmente, el perezoso terrestre, los gliptodontes, algunos caballos, llamas y mamuts se hallan entre estos dos grupos, señalando que estas especies habitaron en zonas con algún grado de cobertura arbórea.

\subsection{TOGUILA}

En esta localidad, valores de $\delta^{13} \mathrm{C}$ para los mamuts van de $-1.3 \%$ a $-5.1 \%$ o con un promedio de $-3.6 \%$ o y para el oxígeno éste fue de $-3.6 \%$ a $-13.3 \%$ con un promedio de $-7.1 \%$. Además, se observa que cuatro mamuts se agrupan a la izquierda, indicando que habitaron en zonas con algún grado de cobertura arbórea y solo uno a la derecha (Tabla 1, Figura 4).

\subsection{VALSEQUILLO}

En la última localidad, se puede observar que en el caso de los caballos su promedio de $\delta^{13} \mathrm{C}$ es de $-0.98 \%$, mientras que para la $\delta^{18} \mathrm{O}$ la media es de $-7.0 \%$. Para los gonfoterios los valores promedio de $\delta^{13} \mathrm{C}$ y $\delta^{18} \mathrm{O}$ son de $-4.2 \%$ y $-7.7 \%$, respectivamente; en cambio las medias de $\delta^{13} \mathrm{C}$ y $\delta^{18} \mathrm{O}$ de los mamuts son de $-1.8 \%$ y $-6.1 \%$, respectivamente. $\mathrm{El}$ carpincho muestra valores isotópicos de carbono de $-2.1 \%$ y de oxígeno de $-6.5 \%$, mientras que el $\delta^{13} \mathrm{C}$ del perezoso terrestre es de $-4.7 \%$ y 


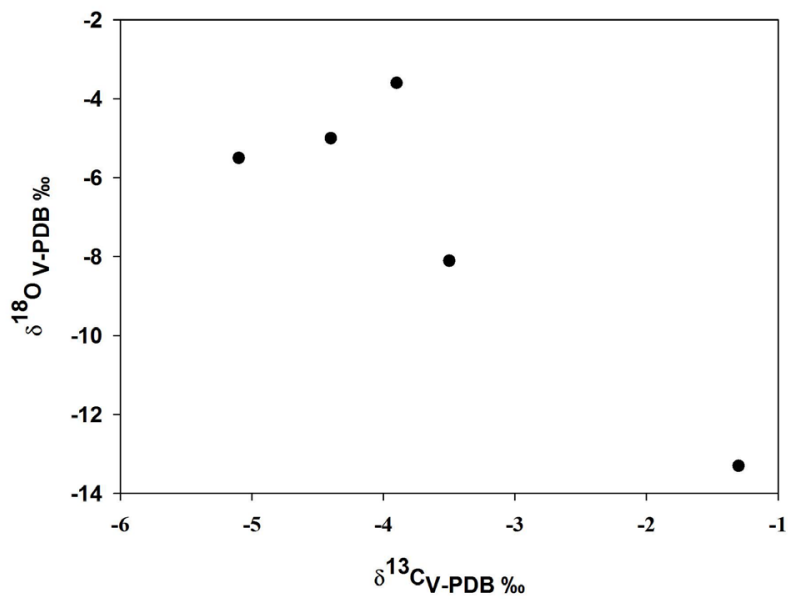

Figura 4 Comparación de los valores isotópicos de carbono y oxígeno de los mamuts de Tocuila. Los valores isotópicos de carbono y oxígeno están expresados en Vienna-Pee-DeeBelemnite y en por mil (V-PDB \%o).

de $\delta^{18} \mathrm{O}-6.5 \%$. El gráfico realizado con los valores de $\delta^{13} \mathrm{C}$ y $\delta^{18} \mathrm{O}$ muestra que la mayor parte de los gonfoterios forman un grupo, mientras el resto de las especies y un gonfoterio se distribuyen en la mitad del gráfico (Tabla 1, Figura 5).

\section{Discusión}

\subsection{PALEOAMBIENTE}

En la Tabla 1 y en las figuras 2 a 5 se puede observar que los valores isotópicos de oxígeno de los animales que habitaron en Rancho La Amapola, Tocuila y Valsequillo son diferentes entre ellos. En cambio, los pertenecientes a la mastofauna del Fin del Mundo son relativamente semejantes. Si los animales migraron, los valores isotópicos de oxígeno hallados en el esmalte dental reflejaran la composición isotópica del oxígeno de las fuentes de agua que bebieron y no las de las condiciones climáticas del sitio donde fueron hallados (Pellegrini et al., 2008).

Además, las diferencias entre los valores isotópicos de oxígeno pueden ser debidas a las diferencias entre la altitud, la latitud y la temperatura de los sitios donde estos animales vivían, los cuales afectan la relación isotópica del oxígeno del agua de la lluvia (Yann et al., 2013).

Los valores isotópicos de carbono así como los gráficos realizados entre los valores de $\delta^{13} \mathrm{C}$ y $\delta^{18} \mathrm{O}$ de cada sitio reflejan tanto el tipo de dieta y de vegetación de cada especie como el ambiente donde ellos habitaron. En el Fin del Mundo, los gonfoterios poseían una dieta mixta $\mathrm{C}_{3} / \mathrm{C}_{4}$ y habitaban en zonas que poseían algunos árboles, mientras que los tapires y mastodontes vivían en zonas boscosas y se alimentaban de plantas $\mathrm{C}_{3}$. En México, Johnson et al. (2006) consideraron que los mastodontes y gonfoterios tenían el mismo tipo de dieta. Sin embargo, los análisis isotópicos y estudios de microdesgaste han revelado que los mastodontes fueron animales que solo consumían plantas $\mathrm{C}_{3}$, como hojas de árboles y frutos, y que habitaban en bosques. Mientras que los gonfoterios fueron animales que podían incluir en su dieta hojas de árboles, frutos y pastos y que residían en bosques o pastizales (Asevedo et al., 2012; Metcalfe, 2017; Smith y DeSantis, 2018). En cambio, estos mismos estudios muestran que los tapires eran especialistas en el consumo de plantas $\mathrm{C}_{3}$ y preferían bosques o selvas para habitar como sus contrapartes actuales (DeSantis, 2011).

La Figura 2 indica que en el Fin del Mundo debió haber existido un bosque con un pastizal. Sin embargo, los mastodontes y el tapir fueron hallados en un nivel estratigráfico debajo al de los gonfoterios, los cuales están fechados entre los 11550 años AP. Esto indica que en una primera etapa, en el Fin del Mundo existieron condiciones más húmedas que las actuales que permitieron el establecimiento de las plantas $\mathrm{C}_{3}$ y de los mastodontes y el tapir. Posteriormente, en una segunda etapa, el bosque fue remplazado por un bosque con pastos, mismo que fue el hábitat de los gonfoterios.

Los análisis de paleosuelos de este sitio revelan que hace 10700 años AP. la vegetación fue mixta $\mathrm{G}_{3}$ / $\mathrm{C}_{4}$ y que existieron condiciones semiáridas. Posteriormente, entre los 10200 y 7750 años AP el am- 


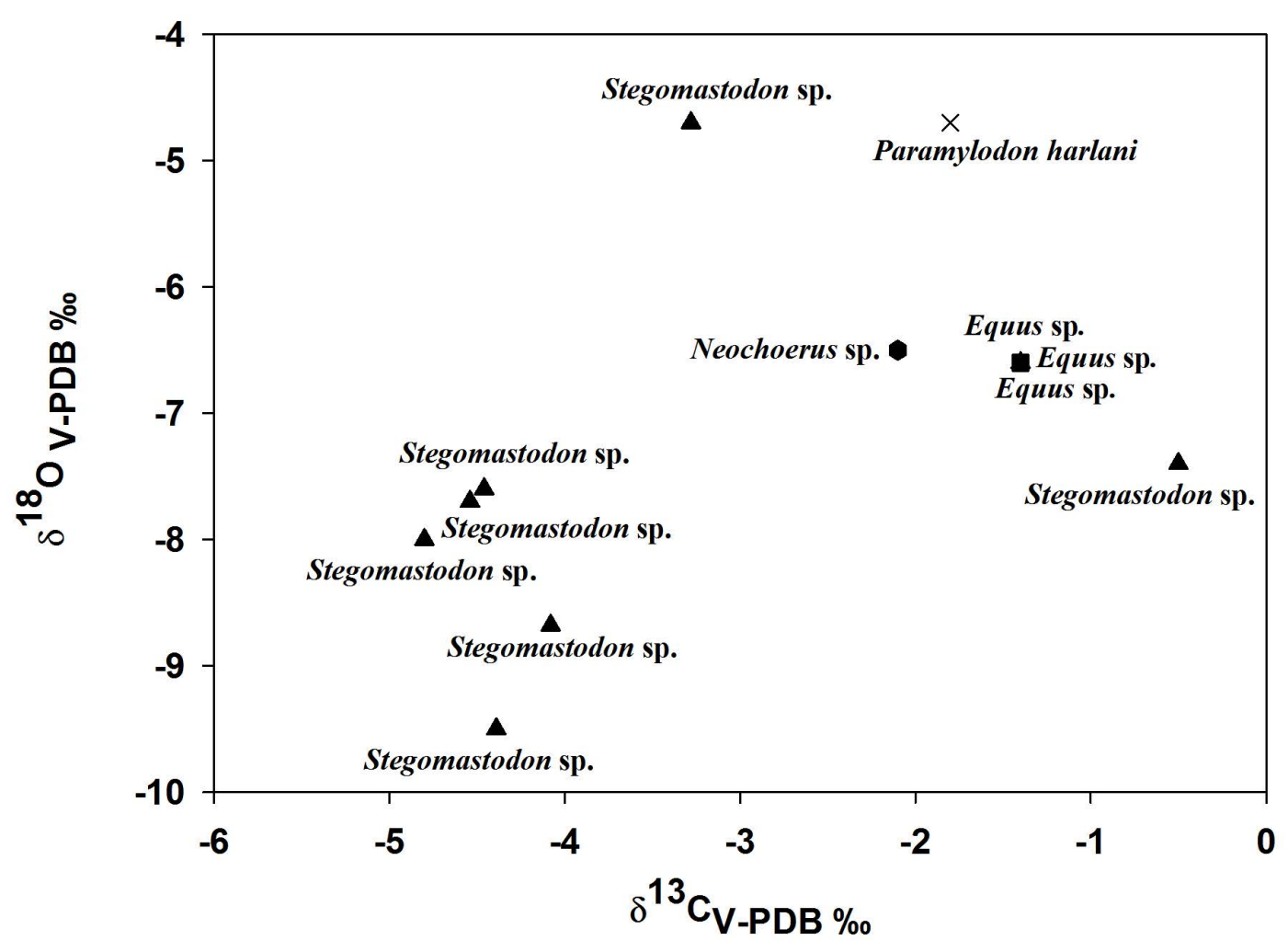

Figura 5 Comparación de los valores isotópicos de carbono y oxígeno de los herbívoros de Valsequillo. Los valores isotópicos de carbono y oxígeno están expresados en Vienna-Pee-Dee- Belemnite y en por mil (V-PDB \%o)

biente se volvió húmedo favoreciendo la presencia de plantas $\mathrm{C}_{3}$ y finalmente hacia los 7000 años $\mathrm{AP}$ se establecieron las condiciones áridas que persisten en la actualidad en el sitio (Cruz-y-Cruz et al., 2016).

Para Rancho La Amapola, la Tabla 1 y la Figura 3 muestran la existencia de animales con diferentes hábitos alimenticios y de hábitat. Así, animales como los tapires y los mastodontes que consumían plantas $\mathrm{C}_{3}$ y que habitaban en bosques coexistían con especies propias de pastizal y que se alimentaban de plantas $\mathrm{C}_{4}$, como los bisontes, los caballos y los mamuts. Johnson et al. (2006) consideraban que muchas especies de herbívoros del Pleistoceno, tales como bisontes, caballos, camellos o mamuts, consumían solo pastos y eran indicadores de pasti- zales y que otros como los berrendos gliptodontes, llamas o perezosos habitaban solo en bosques. Sin embargo, estudios basados en análisis de mesodesgaste, microdesgaste e isótopos estables de carbono y oxígeno muestran que estos animales no eran especialistas en su dieta y hábitat, pudiendo consumir una amplia variedad de plantas y vivir en diversos tipos de vegetación (Rivals y Sempreboon, 2006; Rivals et al., 2007, 2012; Barrón-Ortíz et al., 2014; Marín-Leyva et al., 2015; Pérez-Crespo et al., 2018).

Esto indica que en Rancho La Amapola existieron zonas boscosas y pastizales mismos que permitieron la coexistencia de estos animales en el Pleistoceno tardío. El registro polínico del sitio indica la presencia de árboles, herbáceas y cactáceas (Sán- 
chez-Martínez y Alvarado, 2012). Lo anterior ha llevado a proponer la existencia de un humedal que se desarrolló junto al manantial, así como de un bosque de galería, y cerca de éstos, matorrales o pastizales (Corona-M. 2012; Olivera-Carrasco, 2012). Este mosaico de vegetación sostendría a los diversos herbívoros y carnívoros que habitaron en Rancho La Amapola durante el Pleistoceno tardío. Sin embargo, como lo indica Flores (2012), el clima húmedo que permitió el establecimiento de este tipo de vegetación cambió hacia el Holoceno, haciéndose más cálido lo cual posibilitó el establecimiento del matorral xerófilo que existe en la actualidad.

Los mamuts de Tocuila muestran la presencia de un individuo que se alimentó de plantas $\mathrm{C}_{4} \mathrm{y}$ habitaba en pastizales y cuatro individuos de dieta mixta $\mathrm{C}_{3} / \mathrm{C}_{4}$ que habitaron una zona de vegetación también mixta, lo cual muestra la existencia de un pastizal con algunos árboles (Tabla 1, Figura 4). Diversos estudios palinológicos y análisis de paleosuelos realizados en la Cuenca de México revelan la existencia de bosques en zonas altas y de pastizales y algunos árboles en la parte baja, mismos que se expandían y retraían como consecuencia de las fluctuaciones climáticas de finales del Pleistoceno tardío (Caballero et al., 2010; Cruz-y-Cruz et al., 2016).

En el caso de Valsequillo, se observa que los gonfoterios y un mamut vivían en zonas con algún grado de cobertura arbórea y tenían dietas mixtas $\mathrm{C}_{3} / \mathrm{C}_{4}$ junto con caballos, carpinchos, perezosos terrestres y un mamut que se alimentaban de plantas $\mathrm{C}_{4}$ y habitaban en pastizales, lo que indica que en este sitio la vegetación fue heterogénea (Tabla 1, Figura 5). Cruz-Muñoz et al. (2009), basándose en la presencia de diversas especies de micromamíferos, han sugerido la existencia de un matorral xerófilo con un bosque de coníferas. Los matorrales xerófilos son un tipo de vegetación con poca cobertura arbórea clasificándose como abierta, pero que contienen una gran abundancia de cactáceas (Rzedowski, 1981) cuya vía fotosintética es de tipo CAM. Dado que no es posible distinguir isotópicamente este metabolismo de las vías $\mathrm{C}_{3} \mathrm{y}$ $\mathrm{C}_{4}$, no es posible indicar si alguna de las especies muestreadas de Valsequillo consumían plantas CAM como las cactáceas. Sin embargo, tanto la existencia de otras especies de herbívoros como la información de los datos isotópicos obtenidos por los análisis efectuados en los caballos, el carpincho, los gonfoterios, los mamuts y el perezoso, apoyan la propuesta hecha por Cruz-Muñoz et al. (2009) sobre la vegetación de tipo abierta con un componente arbóreo.

\subsection{IMPLICAGIONES}

En los cuatro sitios arqueo-paleontológicos se desarrollaron condiciones ambientales que permitieron el establecimiento de vegetación heterogénea, misma que sirvió para que diversas especies de mamíferos herbívoros, con hábitos alimentarios y preferencias de hábitat diferentes, habitaran estos sitios y posiblemente fueran penecontemporáneas. Esto debió haber permitido que los primeros pobladores tuvieran acceso a una amplia gama de recursos vegetales y animales. En el caso de estos últimos, su carne pudo ser usada como alimento y sus huesos como herramientas, siendo aprovechados sus cadáveres o cazados en forma activa (Johnson et al., 2006). Por ejemplo, en Tocuila existen huesos modificados de mamut, mientras que en Valsequillo algunos restos óseos de caballos, camellos y gonfoterios poseen marcas de cortes y en el Fin del Mundo existe asociación entre puntas Clovis y gonfoterios (Morett et al., 1998; Sánchez et al., 2014). A pesar de que la asociación entre fauna y hombre ha motivado propuestas sobre extinción de muchas de estas especies en todo el mundo, ya sea por caza y sobreexplotación o por introducción accidental de animales (e.g. perros y ratas) vectores de enfermedades (Martin, 1984; Martin y Steadman, 1999; Lyons et al., 2004; Haynes, 2006; Rothschild y Laub, 2006), aún debemos estudiar con mayor detalle los materiales procedentes de los distintos sitios para tener una mayor certeza al respecto. 


\section{Conclusiones}

El Fin del Mundo, Rancho La Amapola, Tocuila y Valsequillo se caracterizaron por presentar condiciones más húmedas que en la actualidad, lo cual permitió que se establecieran bosques y pastizales, mismos que fueron el hogar de diversas especies de animales. Esta gama de recursos permitió a los primeros pobladores establecerse en tales sitios y dispersarse por el país.

\section{Agradecimientos}

Al Programa de Apoyo a Proyectos de Investigación e Innovación Tecnológica - UNAM (\#IN104017) por su respaldo. A Andrew Somerville por su apoyo en la traducción del resumen. A Mariana Mondini y José Luis Prado por sus comentarios que enriquecieron el presente manuscrito.

\section{Referencias}

Álvarez, T., Ocaña, M.A., Arroyo-Cabrales, J., 2012, Restos de mamífero, en Mirambell, L.E. (coord.), Rancho "La Amapola", Cedral. Un sitio arqueológico-paleontológico pleistocénico-holocénico con restos de actividad humana: México D.F., Colección Interdisciplinaria-Serie Memorias-INAH, 147-194.

Ambrose, S.H., DeNiro, M.J., 1986, The isotopic ecology of East African mammals: Oecologia, 69, 395-406.

Andrade, J.L., de la Barrera, E., Reyes-García, C., Ricalde, M.F., Vargas-Soto, G., Cervera, C.J., 2007, El metabolismo ácido de las crasuláceas: diversidad, fisiología ambiental y productividad: Boletín de la Sociedad Botánica de México, 87, 37-50.
Arroyo-Cabrales, J., Polaco, O.J., Johnson, E., Ferrusquía-Villafranca, I., 2010, A perspective on mammalian biodiversity and zoogeography in the Late Pleistocene of México: Quaternary International, 212, 187-197.

Asevedo, L., Winck, G.R., Mothe, D., Avilla, L.S., 2012, Ancient diet of the Pleistocene gomphothere Niomastodon platensis (Mammalian: Proboscidea, Gomphotheriidae) from lowland midlatitudes of South America: Stereomicrowear and tooth calculus analyses combined: Quaternary International, 255, 42-52.

Barrón-Ortíz, C.R., Theodor, J., Arroyo-Cabrales, J., 2014, Dietary resource partitioning in the late Pleistocene horses from Cedral, northcentral Mexico: evidence from the study of dental wear: Revista Mexicana de Ciencias Geológicas, 31, 260-269.

Caballero, M., Lozano-García, S., VázquezSelem, L., Ortega, B., 2010, Evidencias de cambio climático y ambiental en registros glaciales y en cuencas lacustres del Centro de México durante el último máximo glacial: Boletín de la Sociedad Geológica Mexicana, 62, 359-377.

Casar, I., Morales-Puente, P., Cienfuegos, E., Manzanilla, L.R., Otero, F., 2017, Paleodiet Reconstruction based on Carbon and Nitrogen Isotopes of Teeth from Burial in Teopancazco, en Manzanilla, L.R. (ed.), Multiethnicity and Migration at Teopancazco. Investigations of a Teotihuacán Neighborhood Center: Gainesville, Florida, USA, University Press of Florida, 84-118.

Castillo, R., Morales, P., Ramos, S., 1985, El oxígeno-18 en las aguas meteóricas de México: Revista Mexicana de Física, 31, 637-647. 
Cerling, T.E., 1999, Paleorecords of C4 plants and ecosystems, en Sage, R.F., Monson, R.K. (eds.), C4 plant biology: San Diego, USA, Academic Press, 445-469.

Cerling, T.E., Ehleringer, J.R., 2000, Welcome to the C4 world, en Gastaldo, R.A., Dimechele, W.A. (eds.), Phanerozoic terrestrial ecosystems: USA, The Paleontological Society Papers, 6, 273-286.

Chatters, J.C., Arroyo-Cabrales, J., Álvarez, A., Rissolo, D., Luna-Erreguerena, P., 2016, Proyecto Hoyo Negro: Estudio de una humana del Pleistoceno tardío y fauna extinta de Hoyo Negro, Quintana Roo, México, en Jiménez, L.J.C., Serrano, S.C., Aguilar, A.F., González, G.A. (eds.), El poblamiento temprano en América 7 Serie, Prehistoria en América: Ciudad de México, Museo del Desierto, México, 49-69.

Corona-M., E., 2012, Las aves fósiles, en Mirambell, L.E. (coord.), Rancho "La Amapola", Cedral. Un sitio arqueológicopaleontológico pleistocénico-holocénico con restos de actividad humana: México D.F., Golección Interdisciplinaria-Serie Memorias-INAH, 207-223.

Cruz-y-Cruz, T., Pérez-Crespo, V.A., MoralesPuente, P., Sedov, S., Tovar-Liceaga, R.E., Arroyo-Cabrales, J., Terrazas-Mata, A., Sánchez-Miranda, G., 2016, Paleosol (organic matter and pedogenic carbonates) and paleontological $\delta^{13} \mathrm{C}$ records applied to the paleoecology of Late Pleistocene -Holocene in Mexico: Quaternary International, 418, 147-164.

Cruz-Muñoz, V., Arroyo-Cabrales, J., Graham, R.W., 2009, Rodents and lagomorphs (Mammalian) from the Late-Pleistocene deposits at Valsequillo, Puebla, México: Current Research in the Pleistocene, 26, 147-149.

Dansgaard, W., 1964, Stable isotopes in precipitation: Tellus, 16, 436-468.
DeSantis, L.R.G., 2011, Stable isotope ecology of extant tapirs from the Americas: Biotropica, 43, 746-754.

Drucker, D.G., Bocherens, H., 2009, Carbon stable isotopes of mammal bone as tracer of canopy development and habitat use in temperate and boreal contexts, en Creigthon, J.D., Roney, P.J. (eds.), Forests canopies: Forest production, ecosystem health, and climate conditions: New York., USA, Nova Science Publisher Inc., 2-8.

Ehleringer,J.R., Monson, R.L., 1993, Evolutionary and ecological aspects of photosynthetic pathway variation: Annual Review of Ecology and Systematics, 24, 41 1-439.

Feranec, R.S., MacFadden, B.J., 2006, Isotopic discrimination of resource partitioning among ungulates in C3-dominated communities from the Miocene of Florida and California: Paleobiology, 32, 191-205.

Flores, D.A., 2012, Cambios paleoclimáticos durante el Pleistoceno-Holoceno en un área semidesértica, Cedral, en Mirambell, L.E. (coord.), Rancho "La Amapola", Cedral. Un sitio arqueológico-paleontológico pleistocénico-holocénico con restos de actividad humana: México D.F., Colección Interdisciplinaria-Serie Memorias-INAH, $87-146$.

Garrison, E., 2016, Techniques in Archaeological Geology: USA, Springer International Publishing, 345.

Grayson, D.K., 1981, A critical view of the use of archaelogical vertebrates in paleoenvironmental reconstruction: Journal of Ethnobiology, 1, 28-38.

Gröcker, D.R., 1997, Stable-isotopic studies on the collagenic and hydroxylapatite components of fossils: Palaeoecological implications: Lethaia, 30, 65-78.

Harris, J.M, Cerling, T.E., 2002, Dietary adaptions of extent and Neogen African suids: Journal of Zoology, 256, 45-64. 
Hart, T.C., 2016, Issues and directions in phytolith analysis: Journal of Archaeological Science, 68, 24-31.

Haynes, G., 2006, Firts-contact megafaunal extinctions in the Americas at the end of Pleistocene, en Jiménez-López, J.C., Polaco, O.J., Martínez-Sosa, G., Hernández-Flores, R. (eds.),2o Simposio Internacional el Hombre Temprano en América: México, Instituto Nacional de Antropología e Historia, 91-106.

Hoppe, K.A.,Amundson, R., Vavra, M.,McGlaran, M.P., Anderson, D.L., 2004, Isotopic analysis of tooth enamel carbonate from modern North American feral horses: implications for paleoenvironmental reconstructions: Palaeogeography, Palaeoclimatology, Palaeoecology, 203, 299-311.

Johnson, E., Arroyo-Cabrales,J., Polaco, O.J., 2006, Climate, environment, and game animal resources of the Late Pleistocene Mexican grassland, en Jiménez, L.J.C., González, S., Pompa y Padilla, J.A., Ortiz, P.F. (coords.), El hombre temprano en América y sus implicaciones en el poblamiento de la Cuenca de México Primer simposio internacional: México, D.F., Colección Científica, INAH, 231-245.

Koch, P.L., 1998, Isotopic reconstruction of past continental environments: Annual Review Earth Planetary Science, 26, 573-613.

Lee-Throp, J., Sponheimer, M. 2007, Homin Paleodiets. The Contribution of Stable Isotopes, en Henke, W., Tattersall, I. (eds.), Handbook fo Paleoanthropology. Volumen III. Phylogeny of Hominids: New York, USA, Springer International Publishing, 289-310.-585.

Lyman, R.L., 2017, Paleoenvironmental reconstruction from Faunal Remains: Ecological Basics and Analytical Assumptions: Journal of Archaeological Research, 315-371.
Lyons, S.K., Smith, F.A., Brown, J.H., 2004, Of mice, mastodons and men: human-mediated extinctions on four continents: Evolutionary Ecology Research, 6, 339-358.

MacFadden, B., Cerling, T.E., 1996, Mammalian herbivore communities, ancient feeding ecology, and carbon isotopes: a 10 millionyear sequence from the Neogene of Florida: Journal of Vertebrate Paleontology, 16, 103-115.

Marín-Leyva, A.H., DeMiguel, D., GarcíaZepeda, M.L., Ponce-Saavedra, J., ArroyoCabrales, J., Schaaf, P., Alberdi, M.T., 2015, Dietary adaptability of Late Pleistocene Equus from West Central Mexico: Palaeogeography, Palaeoclimatology, Palaeoecology, 441, 748-57.

Martin, P.S., 1984, Prehistoric overkiller: The Global Model, en Martin, P.S., Klein, R.G. (eds.), Quaternary extinctions: A Prehistoric revolution: Tucson, USA, University of Arizona Press, 354-403.

Martin, P.S., Steadman, D.W., 1999, Prehistoric Extinctions on Islands and Continents, en MacPhee, R.D.E. (ed.), Extinctions in Near Time: New York, USA, Kluwer Academic/ Plenum Publisher, 17-93.

Medrano, H., Flexas, J., 2000, Fotorrespiración y mecanismos de concentración del dióxido de carbono, in: Azcón-Bieto, J., Talón, M. (eds.), Fundamentos de Fisiología Vegetal: Madrid, España, McGraw-Hill Interamericana, 187-201.

Metcalfe, J.Z., 2017, Proboscideans isotopic compositions provide insight into ancient humans and their environments: Quaternary International, 433, 147-159.

Mirambell, L., 2012, Las excavaciones, en Mirambell, L. (coord.), Rancho "La Amapola". Cedral. Un sitio arqueológicopaleontológico pleistocénico-holocénico con restos de actividad humana.: México, D.F., Instituto Nacional de Antropología e Historia 31-69. 
Morales-Puente, P., Casar, I., Cienfuegos, E., Manzanilla, L.R., Otero, F., 2017, Geographic Origins and Migration Histories of the Teopancazco Population, en Manzanilla, L.R. (ed.), Multiethnicity and Migration at Teopancazco. Investigations of a Teotihuacán Neighborhood Center: Gainesville, Florida, USA, University Press of Florida, 119-142.

Morett-A., L., Arroyo-Cabrales, J., Polaco, O.J., 1998, Tocuila, a Remarkable Mammoth Site in the Basin of Mexico: Current Research in the Pleistocene, 15, 118-20.

Neumann, K., Chevalier, A., Vrydaghs, L., 2017, Phytoliths in Archaeology: recent advances: Vegetation History and Archaeobotany, 26, 1-3.

Ochoa-Castillo, P., Pérez-Campa, M., Martín del Pozzo, A.L., Arroyo-Cabrales, J., 2003, New excavations in Valsequillo, Puebla, México: Current Research in the Pleistocene, 20, 61-62.

O'Leary, M.H., 1981, Carbon isotope fractionation in plants: Phytochemistry, 2, 553-567.

Olivera-Carrasco, M.T., 2012, Moluscos continentales de Cedral, un sitio del Pleistoceno final de México, en Mirambell, L.E. (coord.), Rancho "La Amapola", Cedral. Un sitio arqueológico-paleontológico pleistocénico-holocénico con restos de actividad humana: México D.F., Colección Interdisciplinaria-Serie Memorias-INAH, 225-283.

Pellegrini, M., Donahue, R.E., Chenery, C., Evans, J., Lee-Throp, J., Montgomery, J., Mussi, M., 2008, Faunal migration in late-glacial central Italy: implications for human resource exploitation: Rapid Communications in Mass Spectrometry, 22, 1714-1726.

Pérez-Crespo, V.A., Arroyo-Cabrales, J., Benammi, M., Santos-Moreno, A., Morett-A., L., Morales-Puente, P., Cienfuegos-Alvarado, E., 2010, Carbon isotopic values of tooth enamel of Mammuthus columbi from Tocuila, State of México, México: Current Research in the Pleistocene, 27, 188-190,

Pérez-Crespo, V.A., Arroyo-Cabrales, J., AlvaValdivia, L.M., Morales-Puente, P., Cienfuegos-Alvarado, E., Otero, FJ., 2014, La paleodieta de cinco especies de mamíferos herbívoros rancholabreanos de Valsequillo, Puebla: Revista Chilena de Antropología, 30, 76-82.

Pérez-Crespo, V.A., Arroyo-Cabrales, J., Sánchez, G., Holliday, V., Morales-Puente, P., Cienfuegos-Alvarado, E., Otero, FJ., 2016, Información isotópica de los gonfoterios, los mastodontes y el tapir de El Fin del Mundo, un sitio Clovis mexicano, en Jiménez, L.J.C., Serrano, S.G., Aguilar, A.F., González, G.A. (eds.), El poblamiento temprano en América. Serie: Prehistoria en América Museo del Desierto, México, 211-220.

Pérez-Crespo, V.A., Arroyo-Cabrales, J., MoralesPuente, P., Cienfuegos-Alvarado, E., Otero, F.J., 2018, Diet and Habitat of Mesomammals and Megamammals from Cedral, San Luis Potosí, México: Geological Magazine, 155, 674-684.

Rivals, F., Semprebon, G.M., 2006, A comparison of the dietary habits of a large sample of the Pleistocene pronghorn Stockoceros onusrosagris from the Papago Springs Cave in Arizona to the modern Antilocapra americana: Journal of Vertebrate Paleontology, 26, 495-500.

Rivals, F., Solounias, N., Mihlbachler, M.C., 2007, Evidence for geographic variation in the diets of late Pleistocene and early Holocene Bison in North America, and differences from the diets of recent Bison: Quaternary Research, 68, 338-346.

Rivals, F., Semprebon, G., Lister, A., 2012, An examination of dietary diversity patterns in Pleistocene proboscideans (Mammuthus, Paloeoloxodon, and Mammut) from Europe and North America as revealed by dental microwear: Quaternary International, 255, 188-195. 
Rzedowski, J., 1981, Vegetación de México: México, Editorial Limusa.

Rothschild, B.M., Laub, R., 2006, Hyperdisease in the Late Pleistocene: validation of an early 20th century hypothesis: Naturwissenschaften, 93, 557-564.

Sánchez, B., 2005, Reconstrucción del ambiente de mamíferos extintos a partir del análisis isotópico de los restos esqueléticos, in Alcorno, P., Redondo, R., Toledo, J. (eds.), Nuevas técnicas aplicadas al estudio de los sistemas ambientales: los isótopos estables: Madrid, España, Universidad Autónoma de Madrid, 49-64.

Sánchez-Martínez, F., Alvarado,J.L., 2012, Análisis palinológico, in Mirambell, L.E. (coord.), Rancho "La Amapola", Cedral. Un sitio arqueológico-paleontológico pleistocénicoholocénico con restos de actividad humana: México D.F., Colección InterdisciplinariaSerie Memorias-INAH, 285-294.

Sánchez, G., Carpenter, J., 2016, Tracking the first people of Mexico: A review of archaeological record, en Kornfield, M., Huckell, B.B. (eds.), Stones, bones, and profile. Exploring archaeological context, early American
Hunter-Gathers, and bison: Colorado, USA, University Press of Colorado, 75-101.

Sánchez, G., Holliday, V.T., Gaines, E.P., Arroyo-Cabrales, J., Martínez-Tagüeña, N., Kowler, A., Lange, T., Hodgins, G.W.L., Mentzer, S.M., Sánchez-Morales, I., 2014, Human (Clovis)-gomphothere (Cuvieronius sp.) association $\sim 13,390$ calibrated yBP in Sonora, Mexico: Proceedings of the National Academy of Sciences, 111, 10972-10977.

Smith, G.J., DeSantis, L.R.G., 2018, Dietary ecology of Pleistocene mammoths and mastodons as inferred from dental microwear textures: Palaeogeography, Palaeoclimatology, Palaeoecology, 492, 10-25.

van der Merwe, N.J., Medina, E., 1989, Photosynthesis and $13 \mathrm{C} / 12 \mathrm{G}$ ratios in Amazonian rain forest: Geochimica et Cosmochimica Acta, 53, 1091-1094.

Yann, T.L., DeSantis, L.R.G., Haupt, R.J., Romer, J.L., Corapi, S.E., Ettenson, D.J., 2013, The application of and oxygen isotope aridity index to terrestrial paleoenvironmental reconstructions in Pleistocene North America: Paleobiology, 39, 576-590. 